\title{
Tendencias argumentativas en columnas de opinión en dos periódicos de alta circulación en Colombia*
}

\author{
LILIANA PATRICIA GARCÍA ZAMBRANO** \\ MIGUEL ÁNGEL CARO LOPERA***
}

Recepción: 7 de agosto de 2019

Aprobación: 21 de noviembre de 2019

Forma de citar este artículo: García, L.P. \& Caro, M.A. (2020). Tendencias argumentativas en columnas de opinión en dos periódicos de alta circulación en Colombia. Cuadernos de Lingüística Hispánica, (35), 97-110.

https://doi.org/ 10.19053/0121053X.n35.2020.9742

* $\quad$ Este artículo de reflexión deviene de la tesis doctoral "Comprensión de columnas de opinión. Propuesta didáctica dirigida a estudiantes universitarios", que la autora desarrolla actualmente dentro del programa de Doctorado en Ciencias de la Educación de la Universidad del Quindío y Rudecolombia.

** Magíster en Literatura Colombiana y Latinoamericana, de la Universidad del Valle y candidata a doctora en Ciencias de la Educación, Universidad del Quindío, Rudecolombia. Profesora catedrática de la Escuela de Ciencias del Lenguaje de la Universidad del Valle. Correo electrónico: lilianapatriciagarcia@gmail.com bib https://orcid.org/0000-0002-4646-843X

*** Doctor en Ciencias de la Educación, Universidad del Quindío, Rudecolombia. Profesor del Programa de Licenciatura en Literatura y Lengua Castellana, y miembro del grupo de investigación DiLeMa (Didáctica de la Lengua Materna y la Literatura), Universidad del Quindío. Correo electrónico: macaro@uniquindio.edu.co@ https://orcid.org/0000-0002-2263-2362 


\section{Resumen}

Teniendo en cuenta la importancia de los medios de comunicación para la sociedad y del rol de la academia en la formación de sujetos reflexivos y críticos, el presente artículo de reflexión, derivado de una tesis doctoral aún en construcción sobre la enseñanza de la comprensión de textos de tipología argumentativa, se propone identificar, desde un análisis histórico-hermenéutico y en un corpus de más de 543 columnas de opinión, cuya temática gira en torno a la educación superior en Colombia durante el período del posconflicto (2016-2018), las principales tendencias argumentativas que allí se destacan y a las que recurren varios de los autores de los dos más importantes periódicos de circulación masiva en Colombia (El Tiempo y El Espectador). Los resultados de la investigación muestran la predominancia de cierto tipo de argumentos (pragmático, causa-efecto, autoridad, ejemplo y por lo probable) que son analizados a luz de la retórica antigua y la neorretórica; gracias a ellos, los autores buscan ganar la adhesión de los miembros de las comunidades discursivas a los que se dirigen.

Palabras clave: argumentación; columnas de opinión; comprensión lectora; educación superior en Colombia; retórica.

\section{Argumentative Trends in Opinion Columns in Two High Circulation Colombian Newspapers}

\section{Abstract}

Taking into account the importance of the media for society and the role of the academy in the training of reflexive and critical subjects, this article of reflection, derived from a doctoral tesis that is still under construction in the teaching of understanding of texts of argumentative typology, intends to identify, from a historical-hermeneutic análisis and in a corpus of more than 543 opinion columns, whose theme revolves around higher education in Colombia during the post-conflict period (2016-2018), the main argumentative tendencies that stand out there and those used by several of the authors of the two must important newspapers of mass circulation in Colombia (El Tiempo and El Espectador). The results of the investigation show the predominance of certain types of reasonings (pragmatic, cause-effect, authority, example and probable) that are analyzed considering ancient and neo-rhetoric; thanks to them, the authors seek to gain the adhesión of the members of the discursive communities to which they are directed.

Keywords: argumentation; opinion columns; reading comprehension; high education in Colombia; rhetoric. 


\section{Tendances argumentatives des articles d'opinion dans deux journaux Colombiens à forte circulation.}

\section{Résumé}

Eu égard à limportance que la société accorde aux médias et au rôle que l>académie accomplit dans la formation des lecteurs réflexifs et critiques, cet article de réflexion, issu d une thèse de doctorat, encore en préparation, sur lenseignement de la compréhension de textes de typologie argumentative, propose didentifier, à partir dune analyse historicoherméneutique et dans un corpus de 543 articles d opinion, dont le contenu tourne autour de lenseignement supérieur en Colombie pendant la période post-conflit (2016-2018), les principales tendances argumentatives qui s'y mettent en valeur et auxquelles plusieurs journalistes des deux de plus importants journaux à grande circulation en Colombie (" El Tiempo » et « El Espectador») font appel. Les résultats de la recherche montrent la prépondérance de certains types d arguments (pragmatiques, de cause à effet, d autorité, d exemple et de probabilité) qui sont analysés à la lumière de la rhétorique ancienne et de la néo-rhétorique et qui permettent aux journalistes de chercher à gagner l'adhésion des membres des communautés discursives auxquelles ils s`adressent.

Mots-clés: argumentation; articles dopinion; compréhension de lecture; enseignement supérieur en Colombie.

\section{Tendências argumentativas em colunas de parecer em dois jornais de alta circulação na Colômbia}

\section{Resumo}

Levando em conta a importância da mídia para a sociedade e o papel da academia na formação de sujeitos reflexivos e críticos, este artigo de reflexão deriva de uma tese de doutorado ainda em construção sobre o ensino da compreensão de texto de tipologia argumentativa, Propõe-se identificar, a partir de uma análise histórico-hermenêutica e em um corpus de mais de 543 colunas de opinião, cujo tema gira em torno do ensino superior na Colômbia durante o período pós-conflito (2016-2018), as principais tendências argumentativas que se destacam e às quais se baseiam vários dos autores dos dois mais importantes jornais de circulação de massa da Colômbia (El Tiempo e El Espectador). Os resultados da pesquisa mostram a predominância de certos tipos de argumentos (pragmáticos, causa-efeito, autoridade, exemplo e provável) que são analisados à luz da retórica antiga e neo-retórica; graças a eles, os autores buscam obter a adesão dos membros das comunidades discursivas para as quais são direcionados.

Palavras-chave: argumentação; colunas de opinião; compreensão de leitura; ensino superior na Colômbia, retórica. 


\section{Introducción}

Sin duda, los medios de comunicación ejercen influencia en las actividades y decisiones cotidianas. Para Santander (2003) y Masterman (1993), los medios en la actualidad no reflejan la realidad tal cual es, sino que la construyen desde una determinada perspectiva. Este panorama supone un reto para la academia, pues pondera como necesidad inminente el desarrollo de habilidades para la comprensión en todos los niveles que aporten herramientas para la interpretación de los diferentes géneros discursivos y la producción de estos. Esta investigación -aún en construcción-se ocupará de identificar los elementos argumentativos de un corpus de 543 columnas de opinión1, para describir elementos y categorías sobresalientes que permitan más adelante elaborar estrategias didácticas para abordar la comprensión de textos argumentativos en el aula.

En este orden de ideas, el fenómeno observado en los medios nacionales se puede enunciar como la construcción de representaciones de la realidad de acuerdo con intereses de actores sociales y políticos. Lo anterior se traduce en la circulación de información muchas veces parcial 0 arbitraria, que requiere de lecturas críticas y reflexivas en el marco de contextos verificables. Así lo sugieren Paul (1984) y Lipman (1998), quienes afirman que es urgente contribuir a la formación de sujetos que no se limiten al dominio del código alfabético, sino que, además, sean capaces de interpretar y comprender lugares de enunciación, intenciones, juicios de valor, ideologías y representaciones de mundo. En palabras de Cassany (2003, p. 114), actitudes que permitan a la persona participar de modo constructivo en el desarrollo de una comunidad plural, respetuosa y progresista.

\section{La columna de opinión, un género en el corazón de la argumentación}

El género discursivo de la columna de opinión, al servicio del periodismo, recoge una amplia tradición histórica en la cual prevalece su condición argumentativa. Diversos autores (entre ellos, Morán, 1988; Martínez,1983; Abril, 1999; Santamaría \& Casals, 2000; Armañanzas \& Díaz,1996) consideran la columna de opinión como un texto argumentativo de periodicidad y ubicación fijas, en el cual se enjuician diferentes temas, preferiblemente actuales, y que viene redactado con libertad expresiva, con frecuencia por un colaborador fijo, periodista o no. Este género se caracteriza por la responsabilidad enunciativa que asume el autor, lo que supone una estrecha y personal relación entre

1 Columnas de opinión de El Espectador y El Tiempo, periódicos de masiva circulación nacional, cuya temática gira en torno a la educación superior en Colombia durante el período del posconflicto (2016-2018). 
el locutor1 y el discurso. Martínez (1983) asume la columna como artículo razonador, orientador, analítico, enjuiciativo, valorativo, que esgrime su valor en el autor. No en vano, para Herrero (1996, p. 104), la columna de opinión es el género en que la firma se convierte en la medida de valor.

Por otro lado, en este género emergen variedad y libertad, lingüística y retórica, desde la expresión hasta el contenido. Este fenómeno constituye en objeto de análisis al ethos del autor, pues -como bien señalan Santamaría y Casals (2000, p. 302)- en las columnas se trata de extraer datos de la realidad periodística o de sus vivencias y pasarlos por la propia ideología, vestida con un lenguaje intimista y de libertad expresiva. Al respecto, cabe señalar que la columna es un discurso de tipo retórico, en el cual el yo del locutor (ethos) apela a las emociones de los receptores (pathos), con un contenido reflexivo o conocimiento comunicado (logos) que apunta a la eficacia persuasiva de convencer. Precisamente, este hecho o recurso se utiliza por parte de los columnistas a fin de intentar persuadir o convencer al lector, a través del ethos; en tal sentido, son determinantes elementos precisos como el carisma, la actitud y la habilidad del autor para acercarse al público y atraerlo:

El principal recurso retórico de la columna que simultáneamente empezó a perfilarse como elemento característico y configurador de este subgénero es la cualidad que hace al autor de un texto digno de confianza ante sus lectores y la autoridad moral con la que se dirige a ellos. (López, 1996, p. 28)

En medio de esta relación entre el autor y el lector (ethos-pathos) emerge la intención discursiva, acuñada por Herrero (1996, p. 105) como una característica determinante del género, en el sentido de que el columnista, al construir el texto, lo hace pensando en el perfil del lector, que sabe que lee y lo sigue publicación a publicación. Ahora bien, el trabajo de crear una columna de opinión desde una atmosfera de intimidad, complicidad o familiaridad con el lector, pone a prueba la capacidad y la actitud del columnista para emplear en su favor todos sus recursos disponibles, y que bien pueden ser cierto tipo de apelaciones directas al lector, la pregunta, el empleo de un lenguaje popular y cercano, pero además el uso de determinados tipos de argumentos, a través de los cuales busca soportar sus opiniones.

1 Asumimos el concepto de locutor, desde Ducrot, como el responsable de la voz del texto: "Decir que una serie lingüística producida por un locutor constituye un enunciado, equivale a decir, en primer lugar, que este locutor, al producirla, se ha presentado como asumiendo la responsabilidad de la misma" (1984, p. 134). 
Por consiguiente, y para el caso que nos ocupa, la columna como texto valorativo, analítico y orientador, que enjuicia con una determinada finalidad de persuadir 0 convencer, será el campo que nos permitirá identificar, a través de una variedad de argumentos, las posturas y tendencias de los autores en dos periódicos de masiva circulación en Colombia (El tiempo y El Espectador) y en torno a la educación superior durante la época del posconflicto en Colombia (septiembre de 2016 a septiembre de 2018).

\section{Estructuras argumentativas por el camino retórico}

Teniendo en cuenta la definición de la teoría de la argumentación de VegaReñón, según la cual esta es "el estudio y la investigación de los conceptos, modelos y criterios relacionados con la identificación, la construcción, el análisis y la evaluación de argumentos" (2013, p. 55), nos acercaremos a las estructuras argumentativas presentes en las columnas de opinión desde una perspectiva retórica ${ }^{3}$, caracterizada por la preocupación por los procesos de comunicación e influjo interpersonal, en medio del paradigma del discurso persuasivo, mirado desde la eficacia de recursos y estrategias para lograr la adhesión del receptor. En palabras de Vega-Reñón, la perspectiva retórica está "más pendiente de los procesos de inducción de creencias, actitudes o acciones en el destinatario[...] al que hay que persuadir, convencer o mover" (Vega-Reñón, 2013, p. 60).

Desde esta posición, nos interesan dos hechos centrales que emergen de las columnas analizadas y que encuentran su correlato en este aparataje teórico: el primero se relaciona con la dispositio de las columnas y el segundo con los tipos de argumentos que allí se sostienen.

En cuanto al primero, recordemos que la dispositio se inscribe como una de las cinco operaciones retóricas (inventio, dispositio, elocutio, memoria y actio) ${ }^{4}$, gracias a las cuales se construyen los discursos. Esta armazón de corte superestructural da cuenta de un exordio (introducción del texto, por lo regular con la tesis), narratio

3 Vega-Reñón propone un modelo tripartito, en el que se compendian las perspectivas lógica, dialéctica y retórica, que se derivan, a su vez, de sendos trabajos aristotélicos: Analíticos, Tópicos y Retórica, respectivamente. Tales perspectivas vienen correlacionadas por Vega-Reñón con focos centrales, paradigmas y objetivos (2013, pp. 59-64).

4 Al respecto, Cicerón (I, 9) dictaba: "La invención es la acción de pensar cosas verdaderas o símiles a la verdad, que vuelvan probable una causa; la disposición es la distribución, en orden, de las cosas encontradas; la elocución es la acomodación de palabras idóneas y sentencias, de acuerdo con la invención; la memoria es la firme percepción del ánimo de cosas y palabras, de acuerdo con la invención; la pronunciación es la moderación de la voz y del cuerpo, según la dignidad de las cosas y las palabras". 
(contextualización de los hechos que dan origen a la tesis), argumentatio (corazón argumentativo del texto), digressio (parte prescindible del texto que formula excursos 0 paréntesis discursivos fuera del tema central) y peroratio (cierre del texto con efecto en la memoria del lector).

Para el caso del segundo, retomamos las categorías argumentativas que propone Perelman (1997): las de los argumentos cuasilógicos ${ }^{5}$, argumentos basados en la estructura de lo real ${ }^{6}$, argumentos basados en los ejemplos (incluidos la ilustración y el modelo) y argumentos basados en las analogías y la metáfora. A la luz de estas taxonomías generales, de las que se desprenden otras subcategorías ${ }^{7}$, procedemos a presentar los resultados del análisis general de las columnas y a proponer, al menos, un ejemplo representativo que nos describa los rasgos de las tendencias argumentativas más significativas.

\section{Análisis de las columnas a la luz de los conceptos expuestos}

De acuerdo con el primer eje temático antes presentado (el de la dispositio), nos dimos a la tarea de tantear la presencia de los argumentos en exordios, narratios, argumentatios y peroratios. Condensados los hallazgos en la Figura 1, podemos notar que la gran mayoría de las columnas responde a una lógica argumentativa de refutación y toma de posición, especialmente en la parte de la argumentatio, sin dejar de lado que ciertos argumentos también hacen presencia en las otras partes del discurso, en virtud de la brevedad de las mismas y de que, en última instancia, los columnistas -más que en la estructura- se preocupan por el desarrollo de sus ideas. Vale la pena recordar que, en la dispositio, según la retórica antigua, el lugar privilegiado para la disposición de argumentos era, precisamente, la argumentatio. No así el exordio (en el que se depositaba la tesis), la narratio (que ofrecía una prosa de orden expositivo), la digressio (que podía contener excursos de otro orden, como el narrativo) y la peroratio (en la que se regresaba a la tesis y se demostraba su vigencia). En tal sentido, las estadísticas recogidas le dan la razón a esta tendencia primigenia de la argumentación:

5 Sobre los argumentos cuasilógicos, afirma Perelman que "tienen parecido a los razonamientos formales en su apariencia lógica; sin embargo, en estos últimos, es necesario precisar todos los términos utilizados a fin de evitar que existan ambigüedades y que se dé la posibilidad de múltiples interpretaciones (1997, p. 81).

6 En cuanto a los argumentos basados en la estructura de lo real, Perelman subraya que estos presentan tres tipos de nexos. El primero tiene que ver con los nexos de sucesión; el segundo, con los de coexistencia; y el tercero, con los nexos simbólicos y las dobles jerarquías (1997, pp. 78-79).

7 Como las de argumentos pragmáticos, causa-efecto, autoridad, por lo probable, de antimodelo, comparación, contradicción e incompatibilidad, de grado y orden, disociación, definición, división, inclusión, entre otros. 


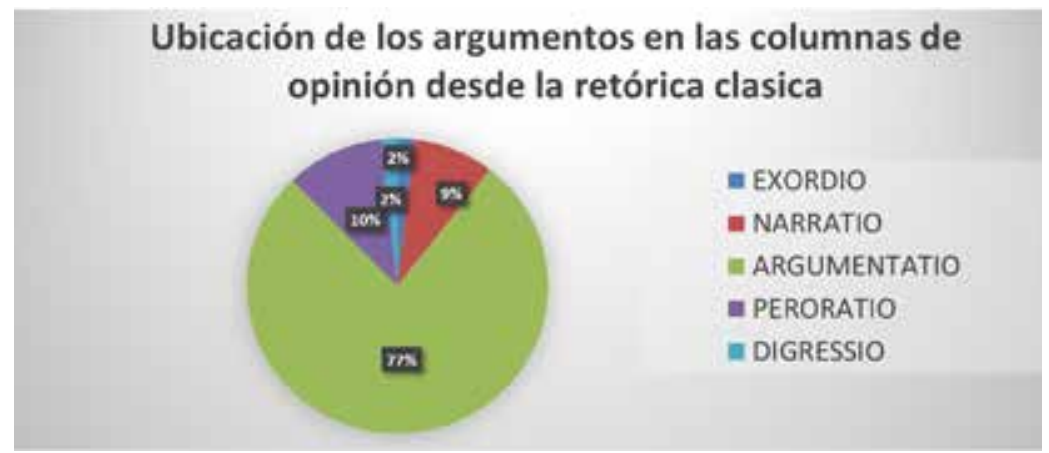

Figra 1. Presencia de argumentos a lo largo de la dispositio de las columnas.

Fuente: elaboración propia

Así las cosas, las columnas revisadas responden, en razón de esto, a la tipología textual argumentativa propia del ámbito social de las "discusiones de problemas sociales controvertidos", en los que se ponen en juego las capacidades lingüísticas del "afianzamiento, refutación y negociación de toma de posición” (Dolz \& Schnewly, 1997, p. 90).

Pasamos ahora al segundo hecho central: el de los argumentos más frecuentes ballados en las columnas de opinión. El resultado se recoge en la siguiente figura:

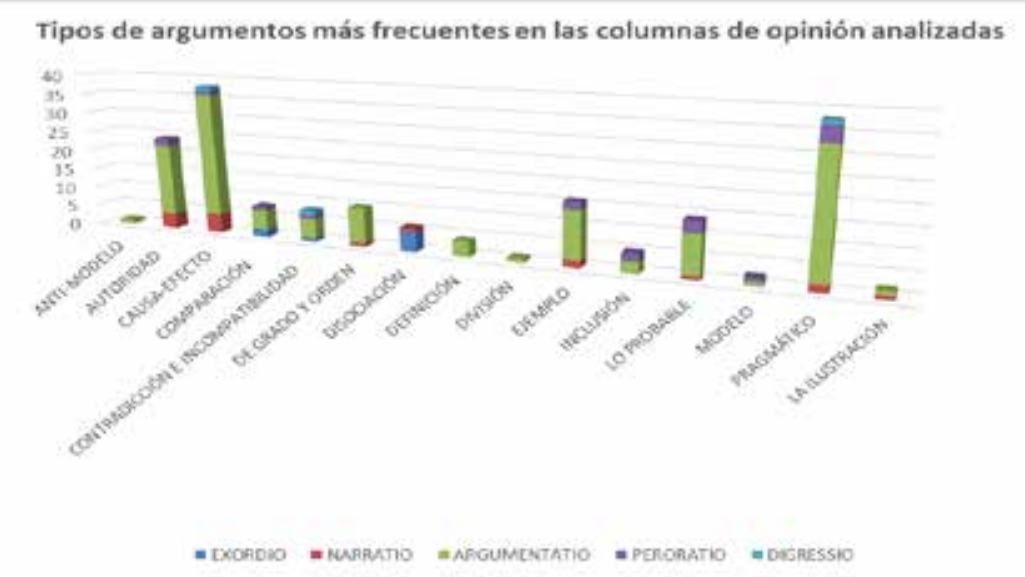

Figura 2. Tipos de argumentos más frecuentes en las columnas de opinión analizadas. Fuente: elaboración propia.

Aquí podemos identificar la presencia dominante de ciertos argumentos, como los de tipo pragmático (21.47\%), seguidos de los de causa-efecto (21.46\%), los de autoridad (13.56 \%), los de ejemplo (9.04\%) y los de lo probable (7.91\%), los cuales se encuentran 
ubicados en su mayoría en la segunda clasificación propuesta por Perelman y que tiene que ver con los argumentos basados en la estructura de lo real. El hecho de que estos argumentos sean los de mayor presencia en la muestra analizada nos lleva a ocuparnos de ellos con cierto detalle, tal como veremos a continuación:

Así pues, el argumento detectado con mayor recurrencia en las columnas de opinión fue el de tipo pragmático (21.47 \%), el cual, señala Perelman (1997), "permite apreciar un acto 0 un acontecimiento con arreglo a sus consecuencias favorables $o$ desfavorables" (p. 115). Y en ese sentido, subraya que las consecuencias pueden ser observadas 0 previstas, seguras 0 presuntas, lo que da lugar a que se piense que este tipo de argumento parece reducir el valor de la causa al de sus consecuencias y deja la impresión de que todos los valores son del mismo orden. Este hecho desempeña un papel esencial en el análisis de los argumentos de tipo pragmático, dado que incluso algunos han querido ver en ello el esquema único que posee la lógica de los juicios de valor, en el sentido de que, para apreciar un acontecimiento, es preciso remitirse a los efectos. Y como muestra de lo anterior, veamos el siguiente ejemplo, tomado de Moisés Wasserman en su texto ¿Qué tan bien lo estamos haciendo? del día 27 de julio de 2018:

La primera parte de la respuesta debe ser un reconocimiento a lo que hemos avanzado durante los últimos decenios. A veces se piensa que mientras menos se reconozca, más se aboga por lo que falta, pero sucede precisamente lo contrario. La falta de reconocimiento rompe la continuidad de buenas iniciativas y desestimula esfuerzos.

El segundo lugar de recurrencia lo ocupa el argumento causa-efecto (21.46\%), el cual, de acuerdo con lo postulado por Perelman (1997), sugiere que: "cuando dos elementos en lo real están asociados entre sí, en un nexo reconocido, es posible fundar sobre este nexo una argumentación que permita pasar de lo que se admite a lo que uno quiere hacer admitir" (p. 113). Vale señalar que en este tipo de argumentos se apela a nexos de sucesión, tales como la relación de causa-efecto en fenómenos o eventos del mismo nivel que se ponen en relación; y de esta manera, a partir de la relación de un nexo causal entre fenómenos, la argumentación puede dirigirse hacia la búsqueda de las causas, hacia la determinación de los efectos y hacia la apreciación de un hecho por sus consecuencias. En este marco de consideraciones, agrega el autor que "cuando se trata de dos actos intencionales, la determinación de la causa se acompaña de la del motivo que ha incitado a realizar el acto" (Perelman, 1997, p. 114). El siguiente ejemplo que hallamos en el texto de José Manuel Restrepo (2017), Universidades públicas vs. Universidades privadas, del día 11 de febrero de 2017, da cuenta de este tipo de nexo causal: 
Estos temas son pertinentes ahora que se hicieron públicos los resultados de un estudio del Banco de la República que evaluó el desempeño de los egresados de la educación superior colombiana, comparando los resultados en función de su condición de provenir de universidades oficiales 0 privadas. El trabajo hace uso de un medio puntual para medir la calidad de la educación superior recibida que corresponde al valor agregado. Este indicador verifica el aporte que realiza la universidad, basado en el avance entre el desempeño del estudiante en las pruebas de entrada a la universidad (examen Saber 11) con respecto a su desempeño en las pruebas de salida (examen Saber Pro). El resultado a que llega es que existe un mejor desempeño en dicho puntual indicador, entre los estudiantes matriculados en universidades oficiales y aquellos matriculados en universidades privadas, muy especialmente en programas de ingeniería, con resultados mucho menos o incluso no contundentes en áreas como medicina, derecho o economía.

En tercer lugar, encontramos el argumento de autoridad (13.56 \%), del cual nos dice Perelman (1997) que se relaciona con el prestigio como cualidad de aquellos que producen en los otros la propensión a imitarlos, y enfatiza que:

Estos son también llamados "autoridades" en determinados asuntos y pueden pertenecer a varias categorías de hombres, entre los cuales sobresalen los sabios, los filósofos, los científicos, entre otros; no obstante, en muchas ocasiones la autoridad también puede ser de tipo impersonal, tal como señala el autor al respecto: “... "la física", "la doctrina", "la religión", "la biblia", etc." (Perelman, 1997, p. 129)

Asimismo, sugiere que la verdadera importancia del argumento por autoridad estriba en "el prestigio de una persona o de un grupo de personas para hacer admitir una tesis" (p. 128), gracias a lo cual, dentro de un discurso sobre cualquier tema, este argumento vendrá como apoyo de otros argumentos; de hecho, aquel que lo use para su beneficio no dejará de valorar la autoridad que concuerda con su tesis, mientras que quien defienda la tesis contraria intentará devaluar constantemente la autoridad que sostiene la tesis del adversario. En tal sentido, el siguiente ejemplo ilustra este tipo de argumento; se trata de la columna El respiro financiero de las universidades estatales: una ilusión fallida, de Adolfo León Atehortúa Cruz, del día 7 de septiembre de 2017:

En efecto, se esperaba que, en virtud del artículo 184 de la Ley 1819 de 2016, reforma tributaria, las universidades públicas recibieran el $40 \%$ de lo recaudado por medio punto del impuesto a las ventas (IVA). Y, así mismo, que por recursos procedentes del impuesto sobre la renta y complementarios (artículo 102), las universidades recibieran el $75 \%$ de 0,4 puntos de dicho tributo, anteriormente denominado CREE. 
Para el cuarto argumento en prevalencia -el de ejemplo (9.04 \%)- Perelman (1997) recuerda que se trata de aquel que pasa de un caso particular a una generalización; aquí se presupone la existencia de algunas regularidades de las que los ejemplos darán una concreción; y aunque, según el autor, lo que podrá ser discutido es el alcance de la regla o el grado de generalización que justifica el caso particular, lo único que no podrá ser discutido es el principio mismo de la generalización. Así pues, en la argumentación por el ejemplo, Perelman subraya que:

[...] a partir de un caso particular se busca la ley, la estructura que él revela o la generalización; se intenta, por medio de la evocación de un número de ejemplos de la misma naturaleza, no dejar ninguna duda respecto al alcance de una situación particular. (1997, p. 143)

El siguiente escrito de Óscar Sánchez, En 2018, eescuelas y universidades en reconciliación, del día 28 de diciembre de 2017, es un ejemplo de este tipo de argumento:

Pero pueden pasar muchas cosas. De hecho, se ha deteriorado la seguridad, están cayendo líderes sociales en todo el país, y la criminalidad está cobrando cada día más vidas. En un ejemplo doloroso, solamente en el sur del Tolima fueron asesinados en los últimos meses la maestra Liliana Astrid Ramírez Martínez, en Coyaima, y el maestro Esnéider Saiz Comba (a cuyas familias, colegas y estudiantes expreso mis condolencias) en Planadas.

Por último, en cuanto al argumento por lo probable $(7.91 \%$ de presencia en la muestra), una característica esencial viene de la argumentación explicativa $y$ cuasilógica, la cual incluye argumentos que buscan dar una apariencia demostrativa de verdad a través de esa búsqueda con la lógica formal, gracias a lo cual se consiguen rápidos efectos de convencimiento en un auditorio particular ${ }^{8}$. Así pues, Perelman (1997) sostiene que el argumento por lo probable alcanza su punto máximo cuando "hay evaluaciones basadas, a la vez, en la importancia de los acontecimientos y en la probabilidad de su aparición, es decir, en la magnitud de las variables y su frecuencia, en la esperanza matemática" (p. 397); por su parte, Martínez (2002) subraya que este se

8 Los argumentos cuasilógicos son argumentos aislados construidos sobre la base de modelos de razonamiento matemático y cercano a la vía explicativa, que generalmente presentan secuencias con propósitos persuasivos que buscan reducir a una lógica más formal los esfuerzos para convencer. Al respecto, Martínez destaca que en la actualidad estos argumentos se utilizan a menudo en las campañas electorales con el fin de movilizar a los votantes a tomar la decisión por un determinado político; asimismo, en su calidad de argumento que apela a las mayorías, también es utilizado de manera masiva en los medios publicitarios de marketing para promover las ventas (2002, p. 185). 
construye "con base en las posibilidades de realización u ocurrencia de un evento o de un comportamiento, y en él se acude a las proporciones y a los porcentajes para lograr credibilidad a través de constataciones empíricas" (p. 185). Un ejemplo de este tipo de argumentos se halla en la columna Los "pilos" desaparecidos, del día 2 de febrero de 2017 y escrita por Luis Carlos Reyes (2017):

Hay casi medio millón de "pilos" desaparecidos que probablemente nunca volvamos a ver como tales. Los desapareció el gobierno -no sólo este sino también los anteriores - al no darles la educación adecuada en preescolar, primaria y bachillerato a la que tenían derecho. Recordemos, claro, que el gobierno es responsabilidad de todos, y por lo tanto también nosotros como sociedad somos responsables.

\section{Conclusiones}

El anterior análisis nos permite llegar a las siguientes conclusiones:

1. Que en todas las columnas se advierte la tendencia a utilizar los diversos tipos de argumentos en la parte que la dispositio dispone para esto: la argumentatio, dado que es aquí precisamente donde se da la lucha por adherir al otro desde la confrontación, a partir de los ejemplos, las estadísticas, las analogías, las citas de autoridad y los datos finamente hilvanados.

2. Que, para la disposición de los argumentos, los autores han privilegiado cierta tendencia hacia la organicidad, a partir de temas conocidos y cercanos al lector hasta llevarlo a otro terreno más complejo, por medio de argumentos que soportan la tesis desde efectos de mayor emoción y, de suyo, rentables a la hora de ganar la adhesión de sus lectores.

3. Que, tal como se vio en los ejemplos anteriores, la mayoría de los argumentos que utilizan los autores de los dos medios de comunicación más importantes del país (El Tiempo y El Espectador) se encuentran dentro de la segunda categoría de la argumentación expuesta por Perelman (1997) en El imperio retórico, lo que corresponde a los argumentos basados en la estructura de lo real, los cuales permiten pasar de lo que se admite a lo que uno quiere hacer admitir. Estos argumentos, que están basados en paradigmas o estructuras que no se ponen en cuestión, nos permiten inferir una evidente carga persuasiva en las columnas analizadas.

4. Que los principales argumentos hallados en las columnas de opinión (pragmático, $21.47 \%$; causa-efecto, $21.46 \%$; autoridad, $13.56 \%$; ejemplo, $9.04 \%$; y por lo probable, $7.91 \%)$ persiguen ganar la adhesión del auditorio por medio de nexos o relaciones entre 
sí. Para el caso del pragmático, se resaltan las consecuencias habitualmente desfavorables; en el de causa-efecto, se ponderan los nexos de sucesión; en el de autoridad, se descansa la argumentación sobre los hombros del otro y de lo otro; en el de ejemplo, se aspira a la generalización desde casos particulares emblemáticos; y en el de lo probable, se busca convencer desde los efectos abrumadores del dato y sus estadísticas.

Por último, conviene dejar dos anotaciones para las investigaciones que vienen; por una parte, señalar la necesidad de seguir profundizando en el análisis de los diferentes tipos de argumentos que se utilizan desde los medios de comunicación, pues no está por demás recordar que, través de este tipo de textos denominados de libre opinión, se modela y vehicula la opinión pública en general; y por otra parte, hacer énfasis en la necesidad de continuar trabajando desde la teoría de la argumentación hacia una actitud más crítica, reflexiva y propositiva, que permita desarrollar en los estudiantes las competencias mediática y argumentativa.

\section{Referencias}

Abril, N. (1999). Periodismo de opinión: claves de la retórica periodística. Madrid: Síntesis.

Armañanzas, E. \& Díaz, J. (1996). Periodismo y argumentación. Géneros de opinión. Bilbao: Editorial Universidad del País Vasco.

Atehortúa, L. (2017, 7 sep.). El respiro financiero de las universidades estatales: una ilusión fallida. El Espectador. Recuperado de https://www.elespectador.com/opinion/el-respirofinanciero-de-las-universidades-estatales-una-ilusion-fallida-columna-712139, del día 7 de septiembre de 2017

Cassany, D. (2003). Aproximaciones a la lectura crítica: teoría, ejemplos y reflexiones. Revista Tarbiya, (32), 113-132.

Cicerón, M. T. (1997). De la invención retórica. México: UNAM.

Dolz, J. \& Schneuwly, B. (1997). Géneros y progresión en expresión oral y escrita. Elementos de reflexión a partir de una experiencia realizada en la Suiza francófona. Textos de Didáctica de la Lengua y de la Literatura, (11), 77-98.

Ducrot, 0. (1984). El decir y lo dicho. Buenos Aires: Hachette.

Herrero, C. (1996). Periodismo político y persuasión. Madrid: Actas.

Lipman, M. (1998). Pensamiento complejo y educación. Madrid: Ediciones de la Torre.

López, F. (1996). La columna periodística. Teoría y práctica el caso de Hilo Directo. Pamplona: EUNSA.

Martínez, J. (1983). Curso general de redacción periodística. Barcelona: Mitre. 
Martínez, M. C. (2002). Estrategias de lectura y escritura de textos. Cátedra Unesco. Cali: Editorial Universidad del Valle.

Masterman, L. (1993). La enseñanza de los medios de comunicación. Madrid: Ediciones de la Torre.

Morán T, E. (1988). Géneros del periodismo de opinión. Crítica, comentario, columna, editorial. Pamplona: Eunsa.

Paul, R. (1984). Critical Thinking: Fundamental to Education for a Free Society. Educational Leadership, (42), 4-14.

Perelman, C. (1997). El imperio retórico. Retórica y argumentación. Santafé de Bogotá: Norma.

Restrepo, M. (2017, 11 feb.). Universidades públicas vs. universidades privadas. El Espectador. Recuperado de https://www.elespectador.com/opinion/columna-42

Reyes, C. (2017, 2 feb.), Los "pilos" desaparecidos. El Espectador. Recuperado de https://www. elespectador.com/opinion/opinion/los-pilos-desaparecidos-columna-677992

Sánchez, 0. (2017, 28 dic.). En 2018, iescuelas y universidades en reconciliación? El Tiempo. Recuperado de http://www.eltiempo.com/opinion/columnistas/oscar-sanchez/en-2018escuelas-y-universidades-en-reconciliacion-165774

Santamaría, L. \& Casals, M. (2000). La opinión periodística. Argumentos y géneros para la persuasión. Madrid: Fragua.

Santander, P. (2009). Analizando los medios y la comunicación: teoría y métodos. Chile: Ediciones Universitarias.

Vega-Reñón, L. (2013). Teoría de la argumentación. En L. Vega-Reñón \& P. Olmos-Gómez (eds.), Compendio de lógica, argumentación y retórica, (pp. 55-66). Madrid: Trotta.

Wasserman, M. (2018, 27 jul.). ¿Qué tan bien lo estamos haciendo? Tenemos educación gratuita, pero eso no impide las grandes diferencias de calidad con la privada. El Tiempo. Recuperado de https:/www.eltiempo.com/opinion/columnistas/moises-wasserman/quetan-bien-lo-estamos-haciendo-248406 